\title{
Collaborative Design Theory and Related Key Technology Study Based on Cloud Computing
}

\author{
Meiren Zhang, Ying Chen \\ School of mathematics and information engineering, Taizhou University, Taizhou, China. \\ Email: zmr@tzc.edu.cn
}

Received 2013

\begin{abstract}
Analyzes the main way of product distribution for collaborative design. According to the requirement of manufacturing collaborative design, apply cloud computing in manufacturing collaborative design and come up the concept of product collaborative cloud design. Study the product collaborative design theory based on cloud computing and the general key technology of cloud computing, semantic web, intelligent matching selection algorithm, STEP and XML technology, version management and conflict resolution arithmetic and so on which related to this theory. The study object of this article is automotive product. Construct a automotive collaborative design system with the key technology to verify the feasibility and validity of the cloud basing collaborative design theory and related technology. This collaborative design system will overcome the weakness that resource and information can not be shared between different department in the same enterprise or different enterprises. Join up this system will help directly enterprise for collaborative design and the repetition construction of collaborative design platform of each enterprise will be avoid. It will reduce the investment of enterprises for constructing and managing collaborative design platform and further reduce the cost of product R\&D with a better and more efficient design.
\end{abstract}

Keywords: Cloud Computing; Cloud Design; Collaborative Design; Semantic Web; Intelligent Matching; Version Management

\section{Introduction}

Along with the increasing competition in manufacturing market, enterprises have to build up self competitive advantage through upgrading rapid reaction ability and enhance innovation capability. Collaborative design is an important research field and application direction of computer supported collaborative work, and it is a specific application of CSCW in CAD. It is a new design methodology to develop new product with parallel collaborative design between different departments located in different places in the internal enterprise. This will help reducing the design cycle, cost and increase the design efficiency to adapt the increasing market competition in manufacturing industry. Cloud design will integrate more design components into internet and more design resource will be shared in internet through design software. The works or elements of each designer, as they wish, can be published, shared and become the design resource of other designers and stored on line. In this way, thousands of designers will become a strong cloud design network with great capacity stock. Cloud design is one of the key technologies of cloud manufacture. This article come up with a product collaborative cloud design theory and applies cloud computing into product collaborative design system.

\section{Problems Existed}

Now most of study to collaborative design focused on concentration and distribution control. But concentrated control can not make different located designers share their design resources and the knowledge can not be reused. Distribution control introduce and apply intellectualization and networking design model represented by semantic net, intelligent Agent, application service provider, manufacturing network etc. it has effected some fields like design knowledge and resource sharing, resource sterilization modeling and capsulation, configuration and scheduling, collaboration and so on. However because of the limitation of type, quantity, ability and usage mode of the provided resource service, the information share based on networking design is not enough, resource unitization ratio is not high, and knowledge reuse and innovation ability in product design is inadequate. In the meantime, every enterprise has to build up their own collaborative design system, the cost of system construction and management is so high that it restricts the 
application extending and development. For instance, some large-scale manufacturing enterprises have already built up the collaborative design system, but the system of each enterprise is independent so it is difficult to proceed with the collaborative design between enterprises.

The research object of this article is automotive product, come up with the cloud basing collaborative design theory, apply cloud computing into collaborative design, make it possible for sharing enterprises' design resources through collaborative design system, reuse the design knowledge, enterprises just need to register in this system then they can start collaborative design between different departments or different enterprises without building up their own collaborative design system to avoid repeated construction and investment, this system is specially a good choice for middle and small-scale enterprises who don't have the foundation to build up their own independent collaborative design system.

\section{Key Technologies Analyzed}

Cloud design build up a frame for manufacturing product collaborative design based on cloud computing and with the cloud manufacturing idea. Manufacture enterprises, especially middle or small- scale enterprises don't need to build up their own collaborative system anymore, just register and access to the system according to their own requirements, and then make use of the system design resources for collaborative design. This will make sharing design resources between enterprises come true and reduce the cost for enterprises to build up collaborative system.

To solve the problem during product manufacturing distribution collaborative, information resources sharing and knowledge representation, searching and reuse, introduce semantic web service into product manufacturing design process, ontology construct for product information and design resources in manufacturing enterprises, represent the semantic in service anabolic process through combination of event-driven service model and semantic service logic diagram.

Study the intelligent disassemble for product design task, intelligent matching for design enterprises and departments and other function; Choosing appropriate matching model and task breaking down to make collaborative design system adapt different changes better in the actual work and find the best design resource. In the meantime reduce the product development cycle, product cost, improve the product quality and increase developing efficiencies.

In the cloud computing environment, design a theory of design documents transition agreement and exchanging rule based on STEP and XML technology. Then the different format design documents can transform and exchange between CAD system and CAM system and
CAD internally and CAM internally in cloud manufacturing. Integrate and share the enterprises product information in the system and enterprises can have fast response to the market change, all of this will help china meet the rapid development requirement of global economy and stand in positive side of global competition.

As different owners of collaborative design will be independent in a limited level meanwhile they have to cooperate with each other, the design conflict will not be avoided. Therefore, detection and resolution of conflict will be very important supporting technology in collaborative design. This paper intends to solve the conflict in the design by version management and version update notification to the design documents.

\section{System Architecture Study of Product Cloud Design}

Based on the study of web collaborative design model represented by ASP service and manufacture grid, integrated application of cloud computing technology and build up new networked collaborative design system, connect different located enterprises or different departments into this system, and these design enterprises could complete each others' advantage by this system, share the resources and technology, and form a fast, good quality, efficiency, low cost design system with high speed. Considering this, here we comes the collaborative design model based on cloud computing for multi enterprises. The framework is described here as followed in Figure 1.

Firstly, enterprises register in the system, add enterprises' information and design resources into system, enterprises can publish design requirements, and cooperated enterprises will download the distributed collaborative task through cloud storage data base server after they $\log$ in the system. When users finish their collaborative design, they can update the design model data into the data base server, the system will then inform other users the version updating information immediately, with this the collaborative design come true. Consumers could also enter into the collaborative design system by web and give their recommendation and product design opinion.

The function of product cloud design system showed below as in Figure 2.

The system identify the semantics according to registered enterprises' information and design resource; after the registered enterprises publish their design requirements, the system will intelligently search and match the best design organization according to different design resources existing in the system and separate the design task. After getting the design task from the system, design organization will cooperate with other design or 


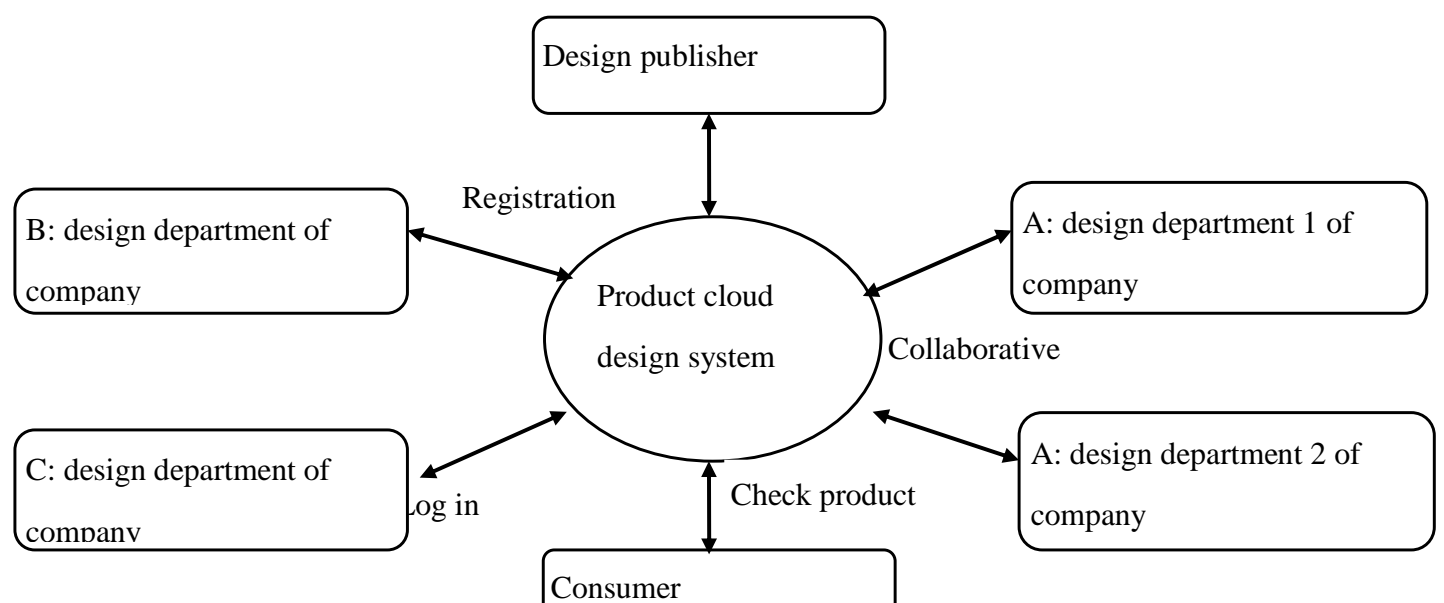

Figure1. System architecture of product cloud design.

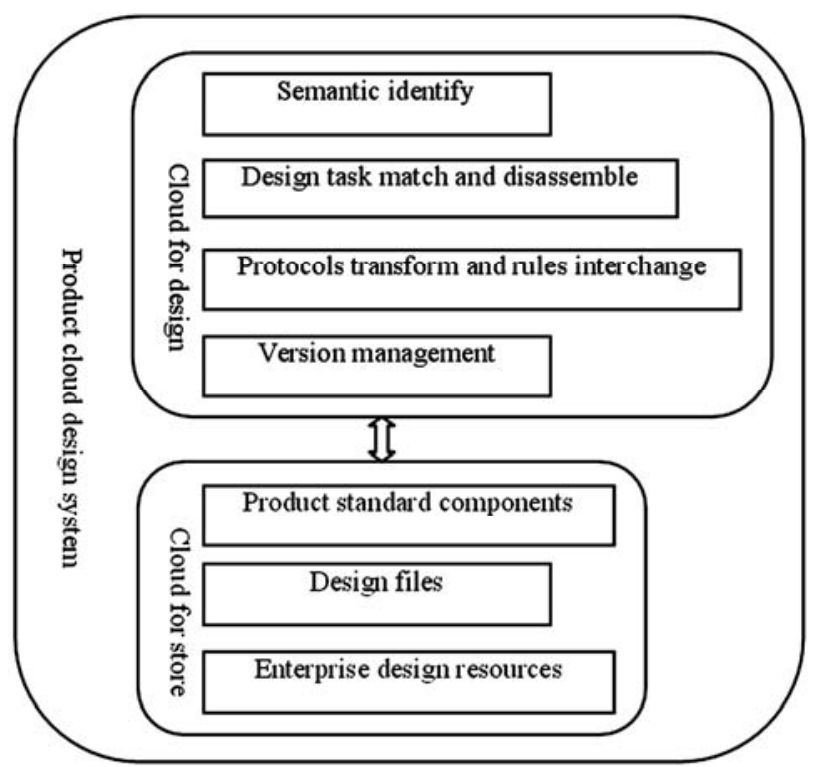

Figure 2. System functions of product cloud design.

ganization to do the collaborative design work with the guidance of the cloud design system. During the design process, different design organization can communicate in time their design documents and design idea. As the CAD software from different design department could be CATIA, UG, Pro/E, Solid works, Cimarron and so on, their file format could be different, the system could use STEP-XML as middleware, according to the established documents transfer agreement change different design documents into XML document which has smaller data volume, with the file transformation regulation, change and transfer the documents between different organization. During the design process, manage the version to solve the version conflict and version traceability. Storage cloud will keep the enterprises' design resources, XML format design documents with different version, and standard components for the product parts and so on.

\section{Ontology Construction and Semantic Annotation of Design Resources}

Ontology description use resource description frame method for cloud design resources and for cloud design process use web ontology language. The data semantic of the design resources and the function semantic annotation of design process will be identified in descriptive language documents.

By building up the cloud design ontology concept system, design resources and design process could be upper-ontology described and realize instantiation by instantiation tools. Design resources could be published in the directory management; design process could be published with web service. Searching and virtual demonstration could be realized by building up virtual server browser with the rich internet application.

\section{Cloud Design Task Intelligent Matching and Disassemble}

Making use of the cloud design searching technology, the key word searching, construction searching, semantic searching and other function can be integrated. When match intelligent semantic, use the method of multi-semantic matching ratio, single service matching and semantic matching between services. When choose and combine services, use the correlated service guide strategy and service filtering algorithm. And realize service choosing with the weighting comprehensive jugging method of parameterized configuration. After choosing the service, combine the service with service process description Petri net extensible markup language and operation process executing language.

\section{Draw up File Transform Protocol and Rule Interchange}

To exchange the design files internally or externally in 
enterprises, the main issue is how to realize exchanging between STEP data and XML data. After intensive study of STEP standard and XML language in the environment of cloud computing, this paper comes up with the thought that exchange enterprises' collaborative design information with STEP-XML standard technology and establish the transition agreement of XML notation of STEP data, and further analyze and realize XML model base of product data exchanging. The following drawing Figure $\mathbf{3}$ describes the way to realize it.

\section{Version Management and Conflict Resolution}

During the product design cycle and product development process, modification for the design can not be avoid, this will generate several different versions, so manage the version generated in design process efficiently will reduce the design cycle and guarantee the design data can be consistent and reuse. This is significant for design cost reducing, design quality improvement and efficiency.

There are two ideas for realization, first is deduction-version management which means save the difference between current version and the one before. When you want use the current version, just add the changed part to the version before, and then you get it. This is an easy way to trace the history of the version and amount of information is small. But the shortcoming is when one version is lost, the entire version behind will be lost in the meantime. The other way is save all different versions with different file names, different versions are independent, they are only get connected by colophon in data base in the format of sheet. With this way, even one version in middle is lost; this will not influence other versions behind. The shortcoming is the amount of information is huge and to save these files will use a lot of resources. This collaborative design system uses the second way as the version management method. Version relationship model is linear structural model.

In the collaborative design process, a large amount of conflicts will happen inevitably. Against the design conflict in design process, a conflict detection method based on relation data base is discussed and target restrain information model for the product is built. And study the way of express the model with XML and relation data base. It also comes up with a conflict detection system construction on the basis of relation data base.

\section{Conclusion}

The study of this paper is based on cloud computing. It studies the Product collaborative design theory and presents a concept of product collaborative design. Also studied the related common key technology and build up a product collaborative design system model based on cloud computing. Integrate design information of resources of enterprises, ontology construction of the product is discussed in the environment of cloud computing and provide the standard and integrated information. Establish the transition agreement between CAD file and XML file in the standard of STEP-XML and based on cloud service. Create XML file which can be transferred and used quickly through network. Intelligently match and separate the design tasks and mange the version and solve the version conflict. In the end, integrate the information between different departments, different enterprises, and between enterprises and consumers, and realize collaborative design.

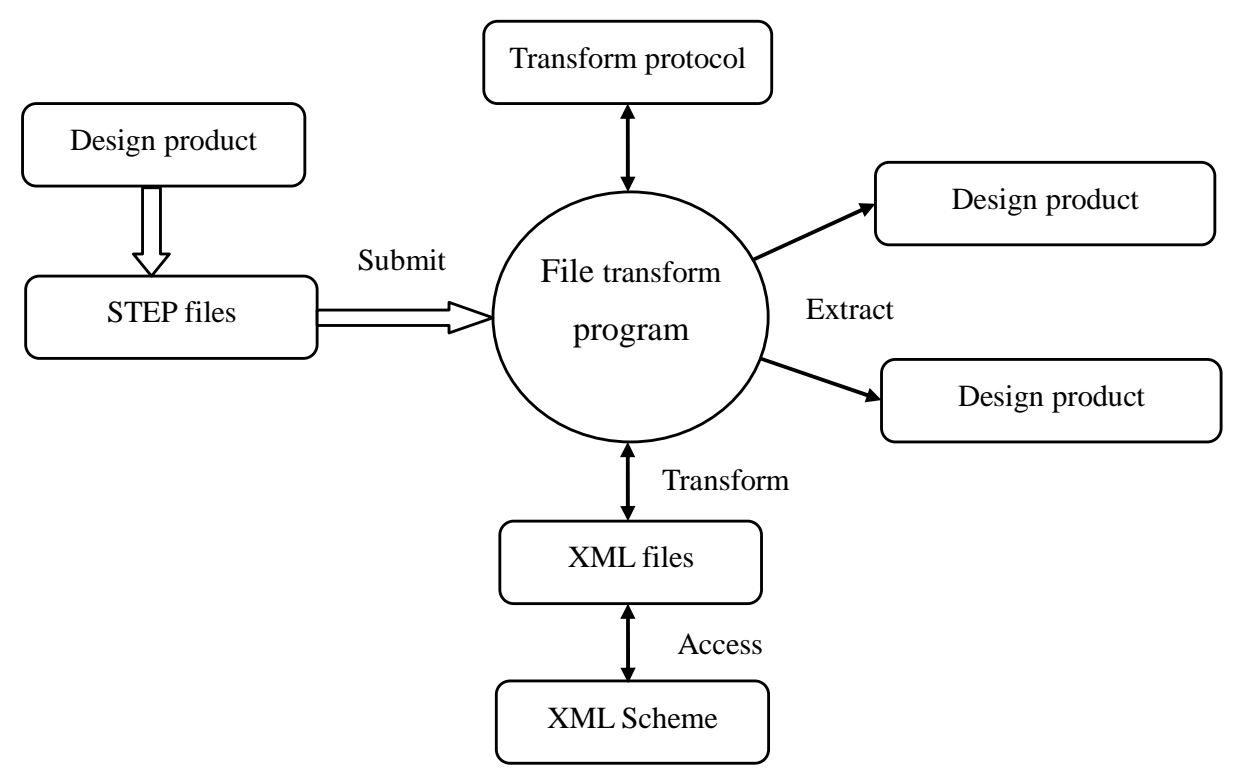

Figure 3. STEP \& XML transform mode. 


\section{Acknowledgment}

A Project Supported by Scientific Research Fund of Zhejiang Provincial Education Department (Y2010 17169).

\section{REFERENCES}

[1] Chenyu. Research on Some Key Technologies for a Regional Networked Manufacturing Platform Based on CSCW[D]. Master's degree thesis, Huazhong University of Science and Technology, Wuhan, China,2004

[2] HE Yan-li, YANG Hai-cheng, HE Wei-ping, MO Rong, JING Shi-kai. Framework of cross-enterprise collaborative manufacturing based on grid theory[J]. Computer Integrated Manufacturing Systems, 2005,11(5):636-639.

[3] Hu Yefa, Tao Fei,Ding Yufeng,Sheng Buyun, Li Yongfeng,Zhou Zude. Study on Framework of Manufacturing Grid Supporting Collaborative Manufacturing[J]. China Mechanical Engineering, 2006, 17(18): 1903-1907.

[4] LI Bo-hu, ZHANG Lin, WANG Shi-long, TAO Fei, CAO Jun-wei, JIANG Xiao-dan, SONG Xiao, CHAI Xu-dong. Cloud manufacturing :a new service-oriented networked manufacturing model[J]. Computer Integrated Manufacturing Systems, 2010,16(1):1-8.

[5] LI Bo-hu. Cloud manufacturing : the cloud computing in manufacturing domain[J].Manufacture Information Engineering of China, 2011,(10):24-26.

[6] CHEN Kang, ZHENG Wei-Min. Cloud Computing: System Instances and Current Research[J]. Journal of Software, 2009, 20(5): 1337-1348.

[7] Amzon, Amzon elastic computer cloud (Amazon) [EB/OL], 2009, 5: http://aws.amazon.com/ec2.

[8] Wang Lizhe, Tao Jie, Kunze M.Scientific clouding: early definition and experience[C].Proceeding 10th IEEE International Conference on High perfComputing and communications .Washington,D.C. IEEE,2008:825-830.

[9] Buyya R, Yeo CS,Venugopal S,et al.Cloudting and emerging IT platforms: vision, hype, and redelivering computing as the 5th uutility[J],Future gecomputer systems, 2009, 25(6):599-616.

[10] Christian de Sainte Marie, A. Paschke, G. Hallmark, et al. RIF Production Rule Dialect W3C Candidate Recommendation. http://www.w3.org/TR/rif-prd/.

[11] H. Boley, M. Kifer. RIF Framework for Logic Dialects W3C Candidate Recommendation. http://www.w3.org/ TR/rif-fld/.

[12] A. Polleres, H. Boley, M. Kifer, et al. RIF Datatypes and Built-Ins 1.0 W3C Candidate Recommendation. http://www.w3.org/TR/rif-dtb/.

[13] Qi Wang. Applied Research of Rules Technology in RDF Application Systems. M.S. thesis (in Chinese), Hohai University, China, 2012.

[14] Wei Chen.The Research of Using RIF to Describe the Semantic Web Rule and Reasoning RIF. M.S. thesis (in Chinese), Jiling University, China, 2010.

[15] O'C. Martin, K. Holger,T. Samson et al. Supporting Rule System Interoperability on the Semantic Web with SWRL. Fourth International Semantic Web Conference (ISWC2005), Galway, Ireland, pp.974-986, 2005.

[16] Xing Wang, Z.M. Ma, et al. RIF Centered Rule Interchange in the Semantic Web. DEXA 2010, Part I, LNCS 6261, pp. 478-486, 2010.

[17] Ian Horrocks, Harold Boley eds. SWRL: A Semantic Web Rule Language Combining OWL and RuleML. http://www.w3.org/Submission/SWRL/\#2.1.

[18] Haifei Zhang, "A Method of Mappings from Reconstructed Relational Databases to RDF Based on RDF View", JDCTA: International Journal of Digital Content Technology and its Applications, Vol. 6, No. 12, pp. 11-19, 2012.

[19] T. Berners-Lee, J. Hendler, O. Lassila. The Semantic Web. Scientific American, vol.284, no.5, pp. 34-43, 2011.

[20] Carstensen P H.Towards information exploration support for engineering designers [EB/OL] (2009-4-27) [201005-15]. http://www.itu.dlk/carstensen/pdf-file/CE97-ex-plo.suppo rt.pdf. 